\title{
On Fuzzy Fundamental Groups and Fuzzy Folding of Fuzzy Minkowski Space
}

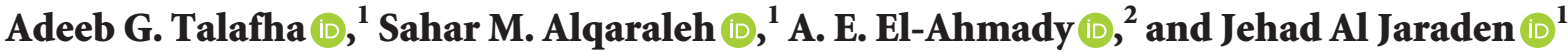 \\ ${ }^{1}$ Department of Mathematics, Faculty of Science, Al-Hussein Bin Talal University, Ma'an, Jordan \\ ${ }^{2}$ Mathematics Department, Faculty of Science, Tanta University, Tanta, Egypt \\ Correspondence should be addressed to Adeeb G. Talafha; adeebgt@ahu.edu.jo
}

Received 8 May 2021; Accepted 21 July 2021; Published 14 August 2021

Academic Editor: Ping-Feng Pai

Copyright (c) 2021 Adeeb G. Talafha et al. This is an open access article distributed under the Creative Commons Attribution License, which permits unrestricted use, distribution, and reproduction in any medium, provided the original work is properly cited.

\begin{abstract}
In this paper, we studied the relations between new types of fuzzy retractions, fuzzy foldings, and fuzzy deformation retractions, on fuzzy fundamental groups of the fuzzy Minkowski space $\tilde{M}^{4}$. These geometrical transformations are used to give a combinatorial characterization of the fundamental groups of fuzzy submanifolds on $\widetilde{M}^{4}$. Then, the fuzzy fundamental groups of the fuzzy geodesics and the limit fuzzy foldings of $\widetilde{M}^{4}$ are presented and obtained. Finally, we proved a sequence of theorems concerning the isomorphism between the fuzzy fundamental group and the fuzzy identity group.
\end{abstract}

\section{Introduction}

Recently, fuzzy sets defined by Zadeh [1] have been widely combined to several mathematical fields, such as complex numbers (see Ramot et al. [2, 3]), topological foldings (see $[4,5]$ ), algebra (see Rosenfeld, Dib, Youssef, and El-Ahmady [6-12]), and many more fields. In the last few decades, Rosenfeld [6] started the development of fuzzy group theory by introducing the concept of the fuzzy subgroup of a group. Then, numerous researchers have studied the theory of fuzzy algebra such as Mordeson and Malik [13] and Demirci $[14,15]$; later on, Negoita and Ralescu [16] replaced the interval $[0,1]$ in Rosenfeld's work by an applicable lattice structure. Anthony and Sherwood [17] presented the notion of triangular norm which is used to redefine the fuzzy subgroup.

Numerous researchers tracked the Rosenfeld-Anthony-Sherwood approach in exploring the fuzzy group theory such as Das [18], Anthony and Sherwood $[19,20]$, Sessa [21], and Akgiil [22]. Youssef and Dib [8, 9] announced an innovative approach to define the fuzzy groupoid and fuzzy subgroupoid. This approach appeared in the theory of fuzzy algebra because of the absence of the concepts of the fuzzy universal set and the fuzzy binary operation which were presented by Youssef and Dib in $[8,9]$. The main difference between the Rosenfeld's approach [6] and that of Youssef and Dib $[8,9]$ is the replacement of the $t$ - norm $f$ with a family of comembership functions $\left\{f_{x y}: x, y \in X\right\}$. Therefore, Dib [9] presented the concept of fuzzy space. Zavadskas et al. [23] applied the idea of Minkowski space with ARAS, TOPSIS, and weighted product methods, and they presented a new model to be applied for numerous problems in which the expert's knowledge is needed to make a proper decision. Abu-Saleem [24] introduced a new type of the fundamental group and studied some types of conditional foldings and unfoldings restricted to the elements of the fuzzy fundamental groups. Also, he presented some theorems and corollaries about the fuzzy fundamental groups of the limit of foldings and the variant and invariant of the fuzzy fundamental group under the folding of the fuzzy manifold into itself. Later, Haçat [25] studied fuzzy $\mathrm{H}$-space and fuzzy $\mathrm{H}$-group and shown that a fuzzy deformation retract of a fuzzy loop space is a fuzzy H-group.

The background of this paper is considered as a continuation of the above efforts in following the study of fuzzy groups by Rosenfild's [6], and it starts from the definition of isometric folding map of Riemannian manifolds by 
Robertson [26], who defined this map between two Riemannian manifolds and stated some of its properties such as the continuity property and the property of conservativeness on the length of piecewise geodesic paths.

Depending on this definition of isometric folding maps, El-Ghoul [27] introduced the concept of folding fuzzy graphs and studied its relation with the fuzzy spheres, and then El-Ghoul and Shamara [28] studied the retraction relation between these kinds of folding fuzzy manifolds.

Another achieved progress had been made while studying the folding of fuzzy manifolds through introducing the definitions of isometric folding map into fuzzy retraction, fuzzy foldings, fuzzy deformation, the folding of fuzzy horocycle, and the folding in the fuzzy Lobachevskian space $[29,30]$.

The aim of this paper is to characterize the fuzzy fundamental groups of fuzzy Minkowski space and their isomorphisms, which is to the best of our knowledge not done yet by anyone else and to study new types of fuzzy retractions, fuzzy folding, and fuzzy deformation retract of fuzzy fundamental groups in $\widetilde{M}^{4}$. This aim motivated us to do this paper and to prove a sequence of theorems focused on the isomorphisms on foldings on $\widetilde{M}^{4}$ space. This characterization is very useful because of its wide range of applications, such as the magneto-static atmospheres, the magnetic forces of some manifolds, and so on, which could be found in $[5,8,12,31-33]$.

\section{Materials and Methods}

Our methodology in this paper depends on constructing an isometric folding map on the fuzzy Minkowski space starting from the fuzzy geodesic of the fuzzy Buchdahi space $\widetilde{B}^{4}$ and then obtaining its cylindrical coordinates by computing the retractions of the fuzzy Minkowski space via some geometrical transformations from the view point of Remannian metric of fuzzy Buchdahi space $\widetilde{B}^{4}$. A fuzzy manifold is a manifold with some physical characters represented by the density function $\mu \in[0,1]$.

Definition 1 (see [29-31]). A fuzzy subset $\underset{\sim}{A}, \mu)$ of a fuzzy manifold $(M, \mu)$ is called a fuzzy retraction of $(\underset{\sim}{M}, \mu)$ if there exist a continuous map $\tilde{r}:(\underset{\sim}{A}, \mu) \longrightarrow(\underset{\sim}{A}, \mu)$ such that $\tilde{r}(a, \mu(a)) \longrightarrow(a, \mu(a)), \forall a \in \underset{\sim}{A}, \mu \in[0,1]$.

Definition 2 (see [29-31]). A fuzzy subset $(\underset{\sim}{\bar{M}}, \widetilde{\mu})$ of a fuzzy manifold $(\underset{\sim}{M}, \mu)$ is called fuzzy deformation retract if there exists a fuzzy retraction $\tilde{r}:(\underset{\sim}{M}, \mu) \longrightarrow(\underset{\sim}{M}, \mu)$ and a fuzzy homotopy $\widetilde{\phi}:(\underset{\widetilde{\phi}}{M}, \mu) \times I \longrightarrow \widetilde{(}(\underset{\sim}{M}, \mu)$ such as $\widetilde{\phi}((x, \mu), 0)=$ $(x, \mu)$ and $\widetilde{\phi}((x, \mu), 1)=\widetilde{r}(\widetilde{x}, \mu), \forall x \in M \cdot \widetilde{\phi}((a, \mu), t)=$ $(a, \mu), \forall(a, \mu) \in \bar{\sim}, t \in I, \mu \in[0,1]$ where $\widetilde{r}(a, \mu(a))$ is the retraction mentioned above.
Definition 3 (see [29-31]). A map $\widetilde{\zeta}: \tilde{M}^{4} \longrightarrow \tilde{M}^{4}$ is said to be an isometric folding of fuzzy submanifolds in $\widetilde{M}^{4}$ into itself iff for any piecewise fuzzy geodesic path $\gamma: J \longrightarrow \tilde{M}^{4}$ is the induced path $\widetilde{\zeta}^{\circ} \gamma: \mathrm{J} \longrightarrow \widetilde{\mathrm{M}}^{4}$ of a piecewise fuzzy geodesic and is of the same length as $\gamma$, where $J=[0,1]$.

\section{Results and Discussion}

In this section, we present and prove some theorems and results describing the relation between fuzzy fundamental groups and each of fuzzy folding $\widetilde{S}_{2}^{1} \subset \widetilde{M}^{4}$, limit of fuzzy geodesic, minimal fuzzy retraction, and some other results.

Theorem 1 (see $[4,11,34,35])$. The fuzzy fundamental group of types of fuzzy deformation retracts of $\widetilde{M}^{4}$ is either isomorphic to $\widetilde{Z}$ or its a fuzzy identity group.

Proof. We will show that $\widetilde{S}_{1}^{1}$ and $\widetilde{S}_{2}^{1}$ are the fuzzy deformation retracts of open fuzzy Minkowski space $\tilde{M}^{4}$. Consider the fuzzy Buchdahi space $\widetilde{B}^{4}$. By using fuzzy cylindrical coordinates $z(\eta), r(\eta), \theta(\eta)$, and $t(\eta)$ with fuzzy metric,

$$
\begin{aligned}
\mathrm{d} s^{2}= & -\gamma^{2}\left(\gamma^{-1} \mathrm{~d} r^{2}(\eta)+r^{2}(\eta) \mathrm{d} \theta^{2}(\eta)+r^{2}(\eta) \sin ^{2} \theta(\eta) \mathrm{d} \theta^{2}(\eta)\right) \\
& +p^{-1} \mathrm{~d} t^{2}(\eta),
\end{aligned}
$$

since $\gamma=(1 / 2) \operatorname{Ln}(B C)$; if $B=C$, we have $\gamma=\operatorname{Ln}(B)$, and if $\operatorname{Ln}(B)=1$, then $\gamma=1$, so equation (1) becomes

$$
\begin{aligned}
\mathrm{d} s^{2}= & -\mathrm{d} r^{2}(\eta)-r^{2}(\eta) \mathrm{d} \theta^{2}(\eta)-r^{2}(\eta) \sin ^{2} \theta(\eta) \mathrm{d} \theta^{2}(\eta) \\
& +p^{-1} \mathrm{~d} t^{2}(\eta)
\end{aligned}
$$

which is the fuzzy metric of $\tilde{M}^{4}$. The fuzzy cylindrical coordinates of $\widetilde{M}^{4}$ are given by

$$
\begin{aligned}
& \tilde{x}_{1}=\frac{s_{1}}{1-i} \sin \left(\frac{s_{2}}{1-\operatorname{ir}(\eta)}\right) \cos \left(\frac{s_{3}}{1-\operatorname{ir}(\eta) \sin \theta(\eta)}\right) \\
& \tilde{x}_{2}=\frac{s_{1}}{1-i} \sin \left(\frac{s_{2}}{1-\operatorname{ir}(\eta)}\right) \sin \left(\frac{s_{3}}{1-\operatorname{ir}(\eta) \sin \theta(\eta)}\right) \\
& \tilde{x}_{3}=\frac{s_{1}}{1-i} \cos \left(\frac{s_{2}}{1-\operatorname{ir}(\eta)}\right) \\
& \tilde{x}_{4}=\frac{s_{4}}{1-\sqrt{p-1}}
\end{aligned}
$$

where $s_{1}, s_{2}, s_{3}$, and $s_{4}$ are the constants of integration. Solving the Lagrangian equations, we obtain fuzzy geodesics and retractions in $\widetilde{M}^{4}$ given as the follows: 
a fuzzy hypersphere $\widetilde{S}_{1}^{1}:-\left(\widetilde{x}_{1}\right)^{2}-\left(\widetilde{x}_{2}\right)^{2}-\left(\widetilde{x}_{3}\right)^{2}-\left(\widetilde{x}_{4}\right)^{2}=0, \quad$ on the null cone,

a fuzzy great circle $\widetilde{S}_{1}^{1}:-\left(\widetilde{x}_{1}\right)^{2}-\left(\widetilde{x}_{2}\right)^{2}-\left(\widetilde{x}_{3}\right)^{2}-\left(\widetilde{x}_{4}\right)^{2}=\left(\frac{s_{1}}{1-i}\right)^{2}+\left(\frac{s_{4}}{1-\sqrt{p-1}}\right)^{2}, \quad$ in $\widetilde{M}^{4}$,

a fuzzy great sphere $\widetilde{S}_{1}^{2}:-\left(\widetilde{x}_{1}\right)^{2}-\left(\widetilde{x}_{2}\right)^{2}-\left(\widetilde{x}_{3}\right)^{2}-\left(\widetilde{x}_{4}\right)^{2}=\left(\frac{s_{1}}{1-i}\right)^{2}+\left(\frac{s_{4}}{1-\sqrt{p-1}}\right)^{2}, \quad$ in $\widetilde{M}^{4}$.

The fuzzy deformation retract of $\widetilde{M}^{4}$ is given by $\tilde{\xi}:\left(\widetilde{M}^{4}-\left\{\widetilde{\mu}_{i}\right\}\right) \times[0,1] \longrightarrow\left(\widetilde{M}^{4}-\left\{\widetilde{\mu}_{i}\right\}\right)$, where $\left(\widetilde{M}^{4}-\left\{\widetilde{\mu}_{i}\right\}\right)$ is the open fuzzy Minkowski space $\widetilde{M}^{4}$, while the fuzzy retraction of $\left(\widetilde{M}^{4}-\left\{\widetilde{\mu}_{i}\right\}\right)$ is given by
$\widetilde{R}: \widetilde{M}^{4}-\left\{\tilde{\mu}_{i}\right\} \longrightarrow \widetilde{S}_{1}^{1}, \widetilde{S}_{2}^{1}, \widetilde{S}_{1}^{2}$, and the fuzzy deformation retract of $\left(\widetilde{M}^{4}-\left\{\tilde{\mu}_{i}\right\}\right)$ onto a fuzzy retraction $\widetilde{S}_{1}^{1} \subset \widetilde{M}^{4}$ is given by

$$
\begin{aligned}
\tilde{\xi}(m, c)= & \cos \frac{\pi c}{2}\left\{\frac{s_{1}}{1-i} \sin \left(\frac{s_{2}}{1-\operatorname{ir}(\eta)}\right) \cos \left(\frac{s_{3}}{1-\operatorname{ir}(\eta) \sin \theta(\eta)}\right), \frac{s_{1}}{1-i} \sin \left(\frac{s_{2}}{1-\operatorname{ir}(\eta)}\right) \sin \left(\frac{s_{3}}{1-\operatorname{ir}(\eta) \sin \theta(\eta)}\right),\right. \\
& \left.\frac{s_{1}}{1-i} \cos \left(\frac{s_{2}}{1-\operatorname{ir}(\eta)}\right), \quad \frac{s_{4}}{1-\sqrt{p-1}}\right\}+\sin \frac{\pi c}{2}\{0,0,0,0\},
\end{aligned}
$$

and the fuzzy deformation retract of $\left(\tilde{M}^{4}-\left\{\tilde{\mu}_{i}\right\}\right)$ onto a fuzzy retraction $\widetilde{S}_{2}^{1} \subset \widetilde{M}^{4}$ is given by

$$
\begin{aligned}
\tilde{\xi}(m, c)= & \frac{1-c}{1+c}\left\{\frac{s_{1}}{1-i} \sin \left(\frac{s_{2}}{1-\operatorname{ir}(\eta)}\right) \cos \left(\frac{s_{3}}{1-\operatorname{ir}(\eta) \sin \theta(\eta)}\right), \frac{s_{1}}{1-i} \sin \left(\frac{s_{2}}{1-\operatorname{ir}(\eta)}\right) \sin \left(\frac{s_{3}}{1-\operatorname{ir}(\eta) \sin \theta(\eta)}\right)\right. \\
& \left.\frac{s_{1}}{1-i} \cos \left(\frac{s_{2}}{1-\operatorname{ir}(\eta)}\right), \quad \frac{s_{4}}{1-\sqrt{p-1}}\right\}+c(2 c-1)\left\{0,0, \frac{s_{1}}{1-i}, \frac{s_{4}}{1-\sqrt{p-1}}\right\},
\end{aligned}
$$

and the fuzzy deformation retract of $\left(\tilde{M}^{4}-\left\{\tilde{\mu}_{i}\right\}\right)$ onto a fuzzy retraction $\widetilde{S}_{1}^{2} \subset \widetilde{M}^{4}$ is given by

$$
\begin{aligned}
\tilde{\xi}(m, c)= & \operatorname{Lne}^{(1-c)}\left\{\frac{s_{1}}{1-i} \sin \left(\frac{s_{2}}{1-\operatorname{ir}(\eta)}\right) \cos \left(\frac{s_{3}}{1-\operatorname{ir}(\eta) \sin \theta(\eta)}\right), \frac{s_{1}}{1-i} \sin \left(\frac{s_{2}}{1-\operatorname{ir}(\eta)}\right) \sin \left(\frac{s_{3}}{1-\operatorname{ir}(\eta) \sin \theta(\eta)}\right),\right. \\
& \left.\frac{s_{1}}{1-i} \cos \left(\frac{s_{2}}{1-\operatorname{ir}(\eta)}\right), \quad \frac{s_{4}}{1-\sqrt{p-1}} . .\right\}+\operatorname{Lne}^{c}\left\{\frac{s_{1}}{1-i} \sin \left(\frac{s_{2}}{1-\operatorname{ir}(\eta)}\right), 0, \frac{s_{1}}{1-i} \cos \left(\frac{s_{2}}{1-\operatorname{ir}(\eta)}\right), \quad \frac{s_{4}}{1-\sqrt{p-1}}\right\} .
\end{aligned}
$$

So, $\pi_{1}\left(\widetilde{M}^{4}-\left\{\widetilde{\mu}_{i}\right\}\right) \cong \pi_{1}\left(\widetilde{S}_{1}^{1}\right), \pi_{1}\left(\widetilde{S}_{1}^{1}\right)$ is isomorphic to the fuzzy identity group, $\pi_{1}\left(\widetilde{M}^{4}-\left\{\widetilde{\mu}_{i}\right\}\right) \cong \pi_{1}\left(\widetilde{S}_{2}^{1}\right) \cong \widetilde{Z}$, and $\pi_{1}\left(\widetilde{M}^{4}-\left\{\widetilde{\mu}_{i}\right\}\right) \cong \pi_{1}\left(\widetilde{S}_{1}^{2}\right)$ is isomorphic to the fuzzy identity group.

Corollary 1. The fuzzy fundamental group of the fuzzy deformation retracts of $\widetilde{M}^{4}-\left\{\widetilde{\mu}_{i}\right\}$ induces two chains of fuzzy fundamental groups up and down $\widetilde{M}^{4}-\left\{\widetilde{\mu}_{i}\right\}$.
Theorem 2. The fuzzy fundamental group of the fuzzy folding of $\widetilde{S}_{2}^{1} \subset \widetilde{M}^{4}$ is either isomorphic to $\widetilde{Z}$ or isomorphic to the fuzzy identity group.

Proof. If the fuzzy folding $\widetilde{\zeta}: \widetilde{S}_{2}^{1} \subset \widetilde{M}^{4} \longrightarrow \widetilde{S}_{2}^{1} \subset \widetilde{M}^{4}$ is given by $\widetilde{\zeta}\left(\tilde{x}_{1}, \widetilde{x}_{2}, \widetilde{x}_{3}, \tilde{x}_{4}\right)=\left(\left|\tilde{x}_{1}\right|, \widetilde{x}_{2}, \tilde{x}_{3}, \tilde{x}_{4}\right)$, then the isomorphism between $\widetilde{S}_{2} \subset \tilde{M}^{4}$ into itself will be defined by 
$\widetilde{\zeta}\left(0,0,\left(s_{1} / 1-i\right),\left(s_{4} / 1-\sqrt{p-1}\right)\right)=\left(0,0,\left(s_{1} / 1-i\right)\right.$, $\left.\left(s_{4} / 1-\sqrt{p-1}\right)\right)$; this type of fuzzy folding and any fuzzy folding isomorphic to it will not induce singularity of $\widetilde{S}_{2}^{1} \subset \widetilde{M}^{4}$, so $\pi_{1}\left(\widetilde{S}_{2}^{1} \subset \widetilde{M}^{4}\right) \cong \widetilde{Z}$.

While if the fuzzy folding $\widetilde{\zeta}_{:} \widetilde{S}_{2}^{1} \subset \widetilde{M}^{4} \longrightarrow \widetilde{S}_{2}^{1} \subset \widetilde{M}^{4}$ is given by $\widetilde{\zeta}\left(\widetilde{x}_{1}, \widetilde{x}_{2}, \widetilde{x}_{3}, \widetilde{x}_{4}\right)=\left(\tilde{x}_{1}, \widetilde{x}_{2}, \widetilde{x}_{3},\left|\widetilde{x}_{4}\right|\right)$, then the isomorphism between $\widetilde{S}_{2} \subset \widetilde{M}^{4}$ into itself will be defined by $\widetilde{\zeta}\left(0,0,\left(s_{1} / 1-i\right), \quad\left(s_{4} / 1-\sqrt{p-1}\right)\right)=\left(0,0,\left(s_{1} / 1-i\right), \quad \mid . s_{4} /\right.$ $1-\sqrt{p-1} \mid)$, and then this type of fuzzy folding and any fuzzy folding isomorphic to it will induce singularity of $\widetilde{S}_{2}^{1} \subset \widetilde{M}^{4}$, so $\pi_{1}\left(\widetilde{S}_{2}^{1} \subset \widetilde{M}^{4}\right)$ is isomorphic to the fuzzy identity group which are two chains of the fuzzy pointing up and down the density function $\eta$.
Corollary 2. The fuzzy fundamental group of the fuzzy folding of $\widetilde{S}_{2}^{1} \subset \widetilde{M}^{4}$ induces two chains of fuzzy fundamental groups up and down $\widetilde{S}_{2}^{1} \subset \widetilde{M}^{4}$.

Theorem 3. The fuzzy fundamental group of the limit of the fuzzy foldings of $\widetilde{S}_{1}^{2}$ in $\widetilde{M}^{4}$ is isomorphic to $\widetilde{Z}$.

Proof. Consider the fuzzy great sphere $\widetilde{S}_{1}^{2}$ of two dimension: $-\left(\widetilde{x}_{1}\right)^{2}-\left(\widetilde{x}_{2}\right)^{2}-\left(\widetilde{x}_{3}\right)^{2}+\left(\widetilde{x}_{4}\right)^{2}=\left(s_{1} / 1-i\right)^{2}+$

$\left(s_{4} / 1-\sqrt{p-1}\right)^{2}$ which is geodesic in $\tilde{M}^{4}$; let $\widetilde{\zeta}_{1}: \widetilde{S}_{1}^{2} \subset \widetilde{M}^{4} \longrightarrow \widetilde{S}_{1}^{2} \subset \widetilde{M}^{4}$ be a fuzzy folding; if we define the series of fuzzy folding maps by the following:

$$
\begin{aligned}
& \widetilde{\zeta}_{1}: \widetilde{S}_{1}^{2} \subset \widetilde{M}^{4} \longrightarrow \widetilde{S}_{1}^{2} \subset \widetilde{M}^{4}, \\
& \widetilde{\zeta}_{2}: \widetilde{\zeta}_{1}\left(\widetilde{S}_{1}^{2} \subset \widetilde{M}^{4} \longrightarrow \widetilde{S}_{1}^{2} \subset \widetilde{M}^{4}\right) \text {, } \\
& \widetilde{\zeta}_{3}: \widetilde{\zeta}_{2}\left(\widetilde{\zeta}_{1}\left(\widetilde{S}_{1}^{2} \subset \tilde{M}^{4} \longrightarrow \widetilde{S}_{1}^{2} \subset \tilde{M}^{4}\right)\right) \\
& \widetilde{\zeta}_{n}:\left(\widetilde{\zeta_{n-1}}\left(\widetilde{\zeta_{n-2}}\left(\widetilde{\zeta_{n-3}}\left(\ldots\left(\tilde{\zeta}_{2}\left(\tilde{\zeta}_{1}\left(\tilde{S}_{1}^{2} \subset \widetilde{M}^{4} \longrightarrow \tilde{S}_{1}^{2} \subset \widetilde{M}^{4}\right)\right)\right) \ldots\right)\right)\right)\right),
\end{aligned}
$$

then $\operatorname{Lim}_{n \rightarrow \infty} \widetilde{\zeta}_{n}\left(\widetilde{\zeta}_{n-1}\left(\widetilde{\zeta}_{n-2}\left(\widetilde{\zeta}_{n-3}\left(\ldots\left(\widetilde{\zeta}_{2}\left(\widetilde{\zeta}_{1}\left(\widetilde{S}_{1}^{2} \subset \tilde{M}^{4}\right.\right.\right.\right.\right.\right.\right.$ $\left.\left.\left.\left.\left.\longrightarrow \widetilde{S}_{1}^{2} \subset \widetilde{M}^{4}\right)\right)\right)_{\tilde{T}_{1}}()\right)\right)$ ) is a fuzzy circle $\widetilde{S}_{2}^{1} \subset \widetilde{M}^{4}$ of dimension one, so $\pi_{1}\left(\widetilde{S}_{2}^{1} \subset \widetilde{M}^{4}\right) \cong \widetilde{Z}$.

Theorem 4. Let $\prod_{m}: \widetilde{S}_{1}^{2} \subset \widetilde{M}^{4} \longrightarrow \widetilde{S}_{1}^{2} \subset \widetilde{M}^{4}$ be given by the fuzzy folding $\prod_{m}\left(\widetilde{x}_{1}, \widetilde{x}_{2}, \widetilde{x}_{3}, \widetilde{x}_{4}\right)=\left(\widetilde{x}_{1}, \widetilde{x}_{2}, \widetilde{x}_{3},\left(\left|\widetilde{x}_{4}\right| / m\right)\right)$, and the fuzzy fundamental group of the limit of the fuzzy folding of the fuzzy geodesic $\widetilde{S}_{1}^{2} \subset \widetilde{M}^{4}$ is isomorphic to $\widetilde{Z}$.

Proof. Defining the isometric chain fuzzy folding of the fuzzy geodesic $\widetilde{S}_{1}^{2} \subset \widetilde{M}^{4}$ of two dimension into itself by the following:

$$
\begin{aligned}
& \prod_{1}:\left\{\left(\frac{s_{1}}{1-i} \sin \left(\frac{s_{2}}{1-\operatorname{ir}(\eta)}\right), 0, \frac{s_{1}}{1-i} \cos \left(\frac{s_{2}}{1-\operatorname{ir}(\eta)}\right), \frac{s_{4}}{1-\sqrt{p-1}}\right\}\right. \\
& \longrightarrow\left\{\left(\frac{s_{1}}{1-i} \sin \left(\frac{s_{2}}{1-\operatorname{ir}(\eta)}\right), 0, \frac{s_{1}}{1-i} \cos \left(\frac{s_{2}}{1-\operatorname{ir}(\eta)}\right),\left|\frac{s_{4}}{1-\sqrt{p-1}}\right|\right\},\right. \\
& \prod_{2}:\left\{\left(\frac{s_{1}}{1-i} \sin \left(\frac{s_{2}}{1-\operatorname{ir}(\eta)}\right), 0, \frac{s_{1}}{1-i} \cos \left(\frac{s_{2}}{1-\operatorname{ir}(\eta)}\right),\left|\frac{s_{4}}{1-\sqrt{p-1}}\right|\right\}\right. \\
& \longrightarrow\left\{\left(\frac{s_{1}}{1-i} \sin \left(\frac{s_{2}}{1-\operatorname{ir}(\eta)}\right), 0, \frac{s_{1}}{1-i} \cos \left(\frac{s_{2}}{1-\operatorname{ir}(\eta)}\right),\left|\frac{s_{4} / 1-\sqrt{p-1}}{2}\right|\right\}\right. \\
& \vdots:\left\{\left(\frac{s_{1}}{1-i} \sin \left(\frac{s_{2}}{1-\operatorname{ir}(\eta)}\right), 0, \frac{s_{1}}{1-i} \cos \left(\frac{s_{2}}{1-\operatorname{ir}(\eta)}\right),\left|\frac{s_{4} / 1-\sqrt{p-1}}{m-1}\right|\right\}\right. \\
& \prod_{m} \mid\left\{\left(\frac{s_{1}}{1-i} \sin \left(\frac{s_{2}}{1-\operatorname{ir}(\eta)}\right), 0, \frac{s_{1}}{1-i} \cos \left(\frac{s_{2}}{1-\operatorname{ir}(\eta)}\right),\left|\frac{s_{4} / 1-\sqrt{p-1}}{m}\right|\right\},\right.
\end{aligned}
$$


then $\quad \operatorname{Lim}_{n \longrightarrow \infty} \prod_{m}=\quad\left\{\left(\left(s_{1} / 1-i\right) \sin \left(s_{2} / 1-\mathrm{ir}\right.\right.\right.$ $\left.(\eta)), 0,\left(s_{1} / 1-i\right) \cos \left(s_{2} / 1-\operatorname{ir}(\eta)\right), 0\right\}$, so $-\left(\widetilde{x}_{1}\right)^{2}-\left(\widetilde{x}_{2}\right)^{2}-$ $\left(\widetilde{X}_{3}\right)^{2}+\left(\widetilde{x}_{4}\right)^{2}=\left(s_{1} / 1-i\right)^{2}$ is the fuzzy great circle $\widetilde{S}_{2}^{1} \subset \widetilde{S}_{1}^{2} \subset \widetilde{M}^{4}$ with $\widetilde{x}_{2}=\widetilde{x}_{4}=0$, so $\pi_{1}\left(\widetilde{S}_{2}^{1} \subset \widetilde{M}^{4}\right) \cong \widetilde{Z}$, and the fuzzy fundamental group of types of the limit of fuzzy foldings and any fuzzy manifold homeomorphic to the fuzzy great circle $\widetilde{S}_{2}^{1} \subset \widetilde{S}_{1}^{2} \subset \widetilde{M}^{4}$ is isomorphic to $\widetilde{Z}$.
Theorem 5. If the fuzzy folding $\prod_{m}: \widetilde{M}^{4} \longrightarrow \widetilde{M}^{4}$ is given by $\prod_{m}\left(\widetilde{x}_{1}, \widetilde{x}_{2}, \widetilde{x}_{3}, \widetilde{x}_{4}\right)=\left(\left(\left|\tilde{x}_{1}\right| / m\right),\left(\left|\tilde{x}_{2}\right| / m\right), \widetilde{x}_{3}, \widetilde{x}_{4}\right)$, then the fuzzy fundamental group of the limit of the fuzzy folding of $\widetilde{M}^{4}$ is isomorphic to the fuzzy identity group.

Proof. Defining the isometric chain of fuzzy foldings of $\widetilde{M}^{4}$ into itself by the following:

$$
\begin{aligned}
& \prod_{1}: \frac{s_{1}}{1-i} \sin \left(\frac{s_{2}}{1-\mathrm{ir}(\eta)}\right) \cos \left(\frac{s_{3}}{1-\mathrm{ir}(\eta) \sin \theta(\eta)}\right), \frac{s_{1}}{1-i} \sin \left(\frac{s_{2}}{1-\operatorname{ir}(\eta)}\right) \sin \left(\frac{s_{3}}{1-\operatorname{ir}(\eta) \sin \theta(\eta)}\right), \frac{s_{1}}{1-i} \\
& \cos \left(\frac{s_{2}}{1-\operatorname{ir}(\eta)}\right), \frac{s_{4}}{1-\sqrt{p-1}} \\
& \longrightarrow\left|\frac{s_{1}}{1-i} \sin \left(\frac{s_{2}}{1-\mathrm{ir}(\eta)}\right) \cos \left(\frac{s_{3}}{1-\mathrm{ir}(\eta) \sin \theta(\eta)}\right)\right|,\left|\frac{s_{1}}{1-i} \sin \left(\frac{s_{2}}{1-\operatorname{ir}(\eta)}\right) \sin \left(\frac{s_{3}}{1-\operatorname{ir}(\eta) \sin \theta(\eta)}\right)\right| \\
& \frac{s_{1}}{1-i} \cos \left(\frac{s_{2}}{1-\operatorname{ir}(\eta)}\right), \frac{s_{4}}{1-\sqrt{p-1}} \\
& \prod_{2}:\left|\frac{s_{1}}{1-i} \sin \left(\frac{s_{2}}{1-\operatorname{ir}(\eta)}\right) \cos \left(\frac{s_{3}}{1-\operatorname{ir}(\eta) \sin \theta(\eta)}\right)\right|,\left|\frac{s_{1}}{1-i} \sin \left(\frac{s_{2}}{1-\operatorname{ir}(\eta)}\right) \sin \left(\frac{s_{3}}{1-\operatorname{ir}(\eta) \sin \theta(\eta)}\right)\right|, \\
& \frac{s_{1}}{1-i} \cos \left(\frac{s_{2}}{1-\operatorname{ir}(\eta)}\right), \frac{s_{4}}{1-\sqrt{p-1}} \\
& \longrightarrow\left|\frac{\left(s_{1} / 1-i\right) \sin \left(s_{2} / 1-\mathrm{ir}(\eta)\right) \cos \left(s_{3} / 1-\mathrm{ir}(\eta) \sin \theta(\eta)\right)}{2}\right|,\left|\frac{\left(s_{1} / 1-i\right) \sin \left(s_{2} / 1-\mathrm{ir}(\eta)\right) \sin \left(s_{3} / 1-\operatorname{ir}(\eta) \sin \theta(\eta)\right)}{2}\right|, \\
& \frac{s_{1}}{1-i} \cos \left(\frac{s_{2}}{1-\operatorname{ir}(\eta)}\right), \frac{s_{4}}{1-\sqrt{p-1}} \\
& \prod_{m}:\left|\frac{\left(s_{1} / 1-i\right) \sin \left(s_{2} / 1-\operatorname{ir}(\eta)\right) \cos \left(s_{3} / 1-\operatorname{ir}(\eta) \sin \theta(\eta)\right)}{m-1}\right|,\left|\frac{\left(s_{1} / 1-i\right) \sin \left(s_{2} / 1-\operatorname{ir}(\eta)\right) \sin \left(s_{3} / 1-\operatorname{ir}(\eta) \sin \theta(\eta)\right)}{m-1}\right| \\
& \frac{s_{1}}{1-i} \cos \left(\frac{s_{2}}{1-\operatorname{ir}(\eta)}\right), \frac{s_{4}}{1-\sqrt{p-1}} \\
& \longrightarrow\left|\frac{\left(s_{1} / 1-i\right) \sin \left(s_{2} / 1-\mathrm{ir}(\eta)\right) \cos \left(s_{3} / 1-\mathrm{ir}(\eta) \sin \theta(\eta)\right)}{m}\right|,\left|\frac{\left(s_{1} / 1-i\right) \sin \left(s_{2} / 1-\mathrm{ir}(\eta)\right) \sin \left(s_{3} / 1-\operatorname{ir}(\eta) \sin \theta(\eta)\right)}{m}\right|, \\
& \frac{s_{1}}{1-i} \cos \left(\frac{s_{2}}{1-\operatorname{ir}(\eta)}\right), \frac{s_{4}}{1-\sqrt{p-1}}
\end{aligned}
$$

then $\quad \operatorname{Lim}_{n \rightarrow \infty} \prod_{m}=\left\{0,0, s_{1} / 1-i \cos \quad\left(s_{2} / 1-\operatorname{ir}(\eta)\right)\right.$, $\left.s_{4} / 1-\sqrt{p-1}\right\}, \quad$ so $-\left(\widetilde{x}_{1}\right)^{2}-\left(\tilde{x}_{2}\right)^{2}-\left(\tilde{x}_{3}\right)^{2}+\left(\tilde{x}_{4}\right)^{2}=$ $\left(s_{1} / 1-i\right) \cos -\left(s_{2} / 1-\operatorname{ir}(\eta)\right)+s_{4} / 1-\sqrt{p-1}$ is the fuzzy hypersurface $\tilde{M}_{2}^{1} \subset \tilde{M}^{4}$ with $\tilde{x}_{1}=\tilde{x}_{2}=0$, so $\pi_{1}\left(\tilde{M}_{2}^{1} \subset \tilde{M}^{4}\right)$ is isomorphic to the fuzzy identity group and any fuzzy manifold homeomorphic to $\widetilde{M}_{2}^{1}$. 
Theorem 6. If the fuzzy folding $\prod_{m}: \tilde{M}^{4} \longrightarrow \tilde{M}^{4}$ is given by $\prod_{m}\left(\tilde{x}_{1}, \tilde{x}_{2}, \tilde{x}_{3}, \tilde{x}_{4}\right)=\left(\widetilde{x}_{1} / m, \tilde{x}_{2} / m, \widetilde{x}_{3} / m, \tilde{x}_{4} / m\right)$, then the fuzzy fundamental group of the limit of the fuzzy folding of $\widetilde{M}^{4}$ is the fuzzy identity group.
Proof. Defining the isometric chain of fuzzy foldings of $\tilde{M}^{4}$ into itself by the following:

$$
\begin{aligned}
& \prod_{1}:\left\{\frac{s_{1}}{1-i} \sin \left(\frac{s_{2}}{1-\operatorname{ir}(\eta)}\right) \cos \left(\frac{s_{3}}{1-\operatorname{ir}(\eta) \sin \theta(\eta)}\right), \frac{s_{1}}{1-i} \sin \left(\frac{s_{2}}{1-\operatorname{ir}(\eta)}\right) \sin \right. \\
& \left.\cdot\left(\frac{s_{3}}{1-\operatorname{ir}(\eta) \sin \theta(\eta)}\right), \frac{s_{1}}{1-i} \cos \left(\frac{s_{2}}{1-\operatorname{ir}(\eta)}\right), \frac{s_{4}}{1-\sqrt{p-1}} \ldots\right\} \\
& \longrightarrow\left\{\left|\frac{s_{1}}{1-i} \sin \left(\frac{s_{2}}{1-\operatorname{ir}(\eta)}\right) \cos \left(\frac{s_{3}}{1-\operatorname{ir}(\eta) \sin \theta(\eta)}\right)\right|,\left|\frac{s_{1}}{1-i} \sin \left(\frac{s_{2}}{1-\operatorname{ir}(\eta)}\right) \sin \left(\frac{s_{3}}{1-\operatorname{ir}(\eta) \sin \theta(\eta)}\right)\right|,\right. \\
& \left.\cdot\left|\frac{s_{1}}{1-i} \cos \left(\frac{s_{2}}{1-\operatorname{ir}(\eta)}\right)\right|,\left|\frac{s_{4}}{1-\sqrt{p-1}}\right| \cdots\right\} \\
& \prod_{2}:\left\{\left|\frac{s_{1}}{1-i} \sin \left(\frac{s_{2}}{1-\mathrm{ir}(\eta)}\right) \cos \left(\frac{s_{3}}{1-\mathrm{ir}(\eta) \sin \theta(\eta)}\right)\right|,\left|\frac{s_{1}}{1-i} \sin \left(\frac{s_{2}}{1-\mathrm{ir}(\eta)}\right) \sin \left(\frac{s_{3}}{1-\operatorname{ir}(\eta) \sin \theta(\eta)}\right)\right|\right. \text {, } \\
& \left.\cdot\left|\frac{s_{1}}{1-i} \cos \left(\frac{s_{2}}{1-\operatorname{ir}(\eta)}\right)\right|,\left|\frac{s_{4}}{1-\sqrt{p-1}}\right|\right\} \\
& \longrightarrow\left\{\left|\frac{\left(s_{1} / 1-i\right) \sin \left(s_{2} / 1-\operatorname{ir}(\eta)\right) \cos \left(s_{3} / 1-\operatorname{ir}(\eta) \sin \theta(\eta)\right)}{2}\right|,\left|\frac{\left(s_{1} / 1-i\right) \sin \left(s_{2} / 1-\operatorname{ir}(\eta)\right) \sin \left(s_{3} / 1-\operatorname{ir}(\eta) \sin \theta(\eta)\right)}{2}\right|\right. \\
& \left.\cdot\left|\frac{\left(s_{1} / 1-i\right) \cos \left(s_{2} / 1-\operatorname{ir}(\eta)\right)}{2}\right|,\left|\frac{s_{4} / 1-\sqrt{p-1}}{2}\right|\right\} \\
& \text { : } \\
& \prod_{m}:\left\{\left|\frac{\left(s_{1} / 1-i\right) \sin m\left(s_{2} / 1-\operatorname{ir}(\eta)\right) \cos \left(s_{3} / 1-\operatorname{ir}(\eta) \sin \theta(\eta)\right)}{m-1}\right|,\left|\frac{\left(s_{1} / 1-i\right) \sin \left(s_{2} / 1-\operatorname{ir}(\eta)\right) \sin \left(s_{3} / 1-\operatorname{ir}(\eta) \sin \theta(\eta)\right)}{m-1}\right|,\right. \\
& \left.\cdot\left|\frac{\left(s_{1} / 1-i\right) \cos \left(s_{2} / 1-\operatorname{ir}(\eta)\right)}{m-1}\right|,\left|\frac{s_{4} / 1-\sqrt{p-1}}{m-1}\right|\right\} \\
& \longrightarrow\left\{\left|\frac{\left(s_{1} / 1-i\right) \sin m\left(s_{2} / 1-\operatorname{ir}(\eta)\right) \cos \left(s_{3} / 1-\operatorname{ir}(\eta) \sin \theta(\eta)\right)}{m}\right|,\left|\frac{\left(s_{1} / 1-i\right) \sin \left(s_{2} / 1-\operatorname{ir}(\eta)\right) \sin \left(s_{3} / 1-\operatorname{ir}(\eta) \sin \theta(\eta)\right)}{m}\right|\right. \\
& \left.\cdot\left|\frac{\left(s_{1} / 1-i\right) \cos \left(s_{2} / 1-\operatorname{ir}(\eta)\right)}{m}\right|,\left|\frac{s_{4} / 1-\sqrt{p-1}}{m}\right|\right\} \text {, }
\end{aligned}
$$

then $\operatorname{Lim}_{n \rightarrow \infty} \prod_{m}=\{0,0,0,0\}$, so $-\left(\tilde{x}_{1}\right)^{2}-\left(\tilde{x}_{2}\right)^{2}-\left(\tilde{x}_{3}\right)^{2}+$ $\left(\tilde{x}_{4}\right)^{2}=0$ is the fuzzy point $\tilde{x}_{1}=\tilde{x}_{2}=\tilde{x}_{3}=\tilde{x}_{4}=0$, and the fuzzy fundamental group of a fuzzy point is the fuzzy identity group.
Theorem 7. The fuzzy fundamental group of the end limits of fuzzy foldings of the n-dimensional fuzzy manifold $\widetilde{F}^{n}$ homeomorphic to $\tilde{M}^{n}$ into itself is the fuzzy identity group. 
Proof. Let $\widetilde{\xi}_{1}: \widetilde{F}^{n} \longrightarrow \widetilde{F}^{n}$ be a fuzzy folding of $\widetilde{F}^{n}$ into itself, we have the following chains:

$$
\begin{aligned}
& \widetilde{F}^{n} \stackrel{\dot{\xi}_{1}^{1}}{\longrightarrow} \widetilde{F}_{1}^{n} \stackrel{\dot{\xi}_{2}^{1}}{\longrightarrow} \widetilde{F}_{2}^{n} \stackrel{\dot{\xi}_{3}^{1}}{\longrightarrow} \ldots \widetilde{F}_{n-1}^{n} \stackrel{\operatorname{Lim}_{\longrightarrow} \widetilde{\xi}_{i}^{1}}{\longrightarrow} \widetilde{F}^{n-1}, \\
& \widetilde{F}^{n-1} \stackrel{\dot{\xi}_{1}^{2}}{\longrightarrow} \widetilde{F}_{1}^{n-1} \stackrel{\dot{\xi}_{2}^{2}}{\longrightarrow} \widetilde{F}_{2}^{n-1} \stackrel{\dot{\xi}_{3}^{2}}{\longrightarrow} \ldots \widetilde{F}_{n-1}^{n-1} \stackrel{\operatorname{Lim}_{\infty}}{\longrightarrow} \widetilde{\xi}_{i}^{2} \widetilde{F}^{n-2}, \\
& \widetilde{F}^{n-2} \stackrel{\dot{\xi}_{1}^{3}}{\longrightarrow} \widetilde{F}_{1}^{n-2} \stackrel{\dot{\xi}_{2}^{3}}{\longrightarrow} \widetilde{F}_{2}^{n-2} \stackrel{\widetilde{\xi}_{3}^{3}}{\longrightarrow} \ldots \widetilde{F}_{n-1}^{n-2} \stackrel{\operatorname{Lim}_{i} \widetilde{\xi}_{i}^{3}}{\longrightarrow} \widetilde{F}^{n-3}, \\
& \vdots \\
& \widetilde{F}^{1} \stackrel{\dot{\xi}_{1}^{n}}{\longrightarrow} \widetilde{F}_{1}^{1} \stackrel{\dot{\xi}_{2}^{n}}{\longrightarrow} \widetilde{F}_{2}^{1} \stackrel{\dot{\xi}_{3}^{n}}{\longrightarrow} \cdots \widetilde{F}_{n-1}^{1} \stackrel{i \operatorname{Lim}}{\longrightarrow} \widetilde{\xi}_{i}^{n} \widetilde{F}^{0} .
\end{aligned}
$$

The end of the limits of fuzzy foldings is the zero-dimensional fuzzy manifold which is a fuzzy point, and the fuzzy fundamental group of a fuzzy point is the fuzzy identity group.
Theorem 8. The fuzzy fundamental group of the minimal fuzzy retraction of the $n$-dimensional fuzzy manifold $\widetilde{F}^{n}$ homeomorphic to $\widetilde{M}^{n}$ is the fuzzy identity group.

Proof. Let $\tilde{r_{1}}:\left\{\widetilde{F}^{n}-\widetilde{B}_{j}^{n}\right\} \longrightarrow \widetilde{F}^{n-1}$ be the fuzzy retraction map, we have the following chains:

$$
\begin{aligned}
& \left\{\widetilde{F}^{n}-\widetilde{B}_{j}^{n}\right\} \stackrel{\widetilde{r}_{1}^{1}}{\rightarrow}\left\{\widetilde{F}_{1}^{n}-\widetilde{B}_{1}^{n}\right\} \stackrel{\widetilde{r}_{2}^{1}}{\rightarrow}\left\{\widetilde{F}_{2}^{n}-\widetilde{B}_{2}^{n}\right\} \stackrel{\widetilde{r}_{3}^{1}}{\rightarrow} \cdots\left\{\widetilde{F}_{n-1}^{n}-\widetilde{B}_{n-1}^{n}\right\}^{\operatorname{Lim}_{i \rightarrow \infty} \widetilde{r}_{i}^{1}} \widetilde{F}^{n-1} \\
& \left\{\widetilde{F}^{n-1}-\widetilde{B}_{j}^{n-1}\right\} \stackrel{\widetilde{r}_{1}^{2}}{\rightarrow}\left\{\widetilde{F}_{1}^{n-1}-\widetilde{B}_{1}^{n-1}\right\} \stackrel{\widetilde{r}_{2}^{2}}{\rightarrow}\left\{\widetilde{F}_{2}^{n-1}-\widetilde{B}_{2}^{n-1}\right\} \stackrel{\widetilde{r}_{3}^{2}}{\rightarrow} \cdots\left\{\widetilde{F}_{n-1}^{n-1}-\widetilde{B}_{n-1}^{n-1}\right\}^{\operatorname{Lim}_{i \rightarrow \infty} \widetilde{r}_{i}^{2}} \widetilde{F}^{n-2}, \\
& \left\{\widetilde{F}^{n-2}-\widetilde{B}_{j}^{n-2}\right\} \stackrel{\widetilde{r}_{1}^{3}}{\rightarrow}\left\{\widetilde{F}_{1}^{n-2}-\widetilde{B}_{1}^{n-2}\right\} \stackrel{\widetilde{r}_{2}^{3}}{\rightarrow}\left\{\widetilde{F}_{2}^{n-2}-\widetilde{B}_{2}^{n-2}\right\} \stackrel{\widetilde{r}_{3}^{3}}{\rightarrow} \cdots\left\{\widetilde{F}_{n-1}^{n-2}-\widetilde{B}_{n-1}^{n-2}\right\}^{\operatorname{Lim}_{\rightarrow \infty} \widetilde{r}_{i}^{3}} \widetilde{F}^{n-3}, \\
& \left\{\widetilde{F}^{1}-\widetilde{B}_{j}^{1}\right\} \stackrel{\widetilde{r}_{1}^{n}}{\rightarrow}\left\{\widetilde{F}_{1}^{1}-\widetilde{B}_{1}^{1}\right\} \stackrel{\widetilde{r}_{2}^{n}}{\rightarrow}\left\{\widetilde{F}_{2}^{1}-\widetilde{B}_{2}^{1}\right\} \stackrel{\widetilde{r}_{3}^{n}}{\rightarrow} \cdots\left\{\widetilde{F}_{n-1}^{1}-\widetilde{B}_{n-1}^{1}\right\}^{\operatorname{Lim}_{i \rightarrow \infty} \widetilde{r}_{i}^{n}} \widetilde{F}^{0}
\end{aligned}
$$

So, the minimal fuzzy retraction of the $\mathrm{n}$-dimensional fuzzy manifold $\widetilde{F}^{n}$ coincides with the limit of the fuzzy retractions which is the zero-dimensional fuzzy point space $\widetilde{F}^{0}$, and the fundamental group of a fuzzy point is the fuzzy identity group.
Theorem 9. Let $\widetilde{S}_{1}^{2} \subset \widetilde{M}^{4}$ be the fuzzy deformation retract of $\widetilde{M}^{4}$ and $\widetilde{F}: \widetilde{M}^{4} \longrightarrow \widetilde{M}^{4}$ be the regular fuzzy folding. Then, $\widetilde{\widetilde{F}} \circ \widetilde{D} \cdot \widetilde{R}=\widetilde{D} \cdot \widetilde{R} \circ \widetilde{F}$ and $\pi_{1}\left(\widetilde{F}\left(\widetilde{M}^{4}\right)\right) \cong \pi_{1}\left(\widetilde{F}\left(\widetilde{S}_{1}^{2} \subset \widetilde{M}^{4}\right)\right)$.

Proof. Define the fuzzy deformation retract of $\widetilde{M}^{4}$ as follows: $\tilde{\xi}:\left(\widetilde{M}^{4}-\left\{\widetilde{\mu}_{i}\right\}\right) \times[0,1] \longrightarrow\left(\widetilde{M}^{4}-\left\{\widetilde{\mu}_{i}\right\}\right)$,

$$
\begin{aligned}
\tilde{\xi}(m, c)= & \frac{1-c}{1+c}\left\{\frac{s_{1}}{1-i} \sin \left(\frac{s_{2}}{1-\operatorname{ir}(\eta)}\right) \cos \left(\frac{s_{3}}{1-\operatorname{ir}(\eta) \sin \theta(\eta)}\right), \frac{s_{1}}{1-i} \sin \left(\frac{s_{2}}{1-\operatorname{ir}(\eta)}\right) \sin \left(\frac{s_{3}}{1-\operatorname{ir}(\eta) \sin \theta(\eta)}\right),\right. \\
& \left.\frac{s_{1}}{1-i} \cos \left(\frac{s_{2}}{1-\operatorname{ir}(\eta)}\right), \frac{s_{4}}{1-\sqrt{p-1}}\right\}+\frac{2 c}{1+c}\left\{\frac{s_{1}}{1-i} \sin \left(\frac{s_{2}}{1-\operatorname{ir}(\eta)}\right), 0, \frac{s_{1}}{1-i} \cos \left(\frac{s_{2}}{1-\operatorname{ir}(\eta)}\right), \frac{s_{4}}{1-\sqrt{p-1}}\right\} .
\end{aligned}
$$


Therefore,

$$
\widetilde{\xi}(m, 1)=\left\{\frac{s_{1}}{1-i} \sin \left(\frac{s_{2}}{1-\operatorname{ir}(\eta)}\right), 0, \frac{s_{1}}{1-i} \cos \left(\frac{s_{2}}{1-\operatorname{ir}(\eta)}\right), \frac{s_{4}}{1-\sqrt{p-1}}\right\}=\widetilde{S}_{1}^{2} \subset \tilde{M}^{4} .
$$

If we consider the fuzzy folding as $\widetilde{F}: \widetilde{S}_{1}^{2} \subset \widetilde{M}^{4}$ $\longrightarrow \widetilde{S}_{1}^{2} \subset \widetilde{M}^{4}, \widetilde{F}\left(\widetilde{x}_{1}, \widetilde{x}_{2}, \widetilde{x}_{3}, \widetilde{x}_{4}\right)=\left(\left|\tilde{x}_{1}\right|,\left|\widetilde{x}_{2}\right|,\left|\widetilde{x}_{3}\right|,\left|\widetilde{x}_{4}\right|\right)$, then

$$
\begin{aligned}
& \widetilde{F}\left\{\left(\frac{s_{1}}{1-i} \sin \left(\frac{s_{2}}{1-\operatorname{ir}(\eta)}\right), 0, \frac{s_{1}}{1-i} \cos \left(\frac{s_{2}}{1-\operatorname{ir}(\eta)}\right), \frac{s_{4}}{1-\sqrt{p-1}}\right\}\right. \\
& \quad=\left\{\left(\left|\frac{s_{1}}{1-i} \sin \left(\frac{s_{2}}{1-\operatorname{ir}(\eta)}\right)\right|, 0,\left|\frac{s_{1}}{1-i} \cos \left(\frac{s_{2}}{1-\operatorname{ir}(\eta)}\right)\right|,\left|\frac{s_{4}}{1-\sqrt{p-1}}\right|\right\} .\right.
\end{aligned}
$$
$\left.\left.\tilde{M}^{4}\right)\right)$.

Therefore, $\overline{\widetilde{F}} \circ \widetilde{\mathrm{D}} \cdot \widetilde{\mathrm{R}}=\overline{\widetilde{\mathrm{D}} \cdot \widetilde{\mathrm{R}}} \cdot \widetilde{\mathrm{F}}$ and $\pi_{1}\left(\widetilde{F}\left(\tilde{M}^{4}\right)\right) \cong \pi_{1}\left(\widetilde{F}\left(\widetilde{S_{1}^{2}} \subset\right.\right.$

\section{Conclusion}

We characterized the fuzzy fundamental groups of fuzzy Minkowski space $\tilde{M}^{4}$ and their isomorphisms by defining an isometric folding map on the fuzzy Minkowski space derived from the fuzzy geodesic of the fuzzy Buchdahi space $\widetilde{B}^{4}$; then, we obtained the relation between the concepts: fuzzy folding, fuzzy retraction, fuzzy deformation retraction, and fuzzy fundamental groups of the manifold $\tilde{M}^{4}$. Also, we were able to determine a connection between the limits of the fuzzy foldings and the fuzzy fundamental groups; and finally, we stated and proved the results of this article as a sequence of theorems concerned on the isomorphism between the fuzzy fundamental group and the fuzzy identity group. Our hope in the future is to write the applications of these proved theorems as well as to construct more useful results together with their applications.

\section{Data Availability}

The data used to support the findings of this study have not been made available because the study is theoretical and does not depend on any collected data.

\section{Conflicts of Interest}

The authors declare that there are no conflicts of interest.

\section{Acknowledgments}

This work was kindly supported by Al-Hussein Bin Talal University, Ma'an/Jordan.

\section{References}

[1] L. A. Zadeh, "Fuzzy sets," Information and Control, vol. 8, no. 3, pp. 338-353, 1965.
[2] D. Ramot, R. Milo, M. Friedman, and A. Kandel, "Complex fuzzy sets," IEEE Transactions on Fuzzy Systems, vol. 10, no. 2, pp. 171-186, 2002.

[3] D. Ramot, M. Friedman, G. Langholz, and A. Kandel, "Complex fuzzy logic," IEEE Transactions on Fuzzy Systems, vol. 11, no. 4, pp. 450-461, 2003.

[4] A. E. El-Ahmady, "The deformation retract and topological folding of Buchdahi space," Periodica Mathematica Hungarica, vol. 28, no. 1, pp. 19-30, 1994.

[5] T. Yogalakshmi, E. Roja, and M. K. Uma, "Soft fuzzy soft homotopy and its topological foldings of a soft fuzzy soft manifold," Annals of Fuzzy Mathematics and Informatics, vol. 8, no. 1, pp. 125-139, 2014.

[6] A. Rosenfeld, "Fuzzy groups," Journal of Mathematical Analysis and Applications, vol. 35, no. 3, pp. 512-517, 1971.

[7] K. A. Dip and N. L. Youssef, "Fuzzy Cartesian product, fuzzy relations, and fuzzy functions," Fuzzy Sets and Systems, vol. 41, pp. 299-315, 1991.

[8] N. L. Youssef and K. A. Dib, "A new approach to fuzzy groupoids," Fuzzy Sets and Systems, vol. 49, no. 3, pp. 381-392, 1992.

[9] K. A. Dib, "On fuzzy spaces and fuzzy group theory," Information Sciences, vol. 80, no. 3-4, pp. 253-282, 1994.

[10] K. A. Dib and A. A. M. Hassan, "The fuzzy normal subgroup," Fuzzy Sets and Systems, vol. 98, no. 3, pp. 393-402, 1998.

[11] A. E. El-Ahmady, "Folding and fundamental groups of Buchdahi space," Indian Journal of Science and Technology, vol. 6, no. No. 1, pp. 3940-3945, 2013.

[12] A. E. El-Ahmady, "The variation of the density functions on chaotic spheres in chaotic space-like Minkowski space time," Chaos, Solitons \& Fractals, vol. 31, no. 5, pp. 1272-1278, 2007.

[13] J. N. Mordeson and D. S. Malik, Fuzzy Commutative Algebra, World Scientific, Singapore, Asia, 1998.

[14] M. Demirci, "Smooth groups," Fuzzy Sets and Systems, vol. 117, no. 3, pp. 431-437, 2001a.

[15] M. Demirci, "Smooth subgroups and smooth homomorphisms," Fuzzy Sets and Systems, vol. 117, no. 3, pp. 439-446, 2001b.

[16] C. V. Negoita and D. A. Ralescu, Applications of Fuzzy Sets to System Analysis, Wiley, New York, NY, USA, 1975.

[17] J. M. Anthony and H. Sherwood, "Fuzzy groups redefined," Journal of Mathematical Analysis and Applications, vol. 69, no. 1, pp. 124-130, 1979. 
[18] P. S. Das, "Fuzzy groups and level subgroups," Journal of Mathematical Analysis and Applications, vol. 84, no. 1, pp. 264-269, 1981.

[19] J. M. Anthony and H. Sherwood, "A characterization of fuzzy subgroups," Fuzzy Sets and Systems, vol. 7, no. 3, pp. 297-305, 1982.

[20] H. Sherwood, "Products of fuzzy subgroups," Fuzzy Sets and Systems, vol. 11, no. 1-3, pp. 79-89, 1983.

[21] S. Sessa, "On fuzzy subgroups and fuzzy ideals under triangular norms," Fuzzy Sets and Systems, vol. 13, no. 1, pp. 95-100, 1984.

[22] M. Akgiil, "Some properties of fuzzy groups," Journal of Mathematical Analysis and Applications, vol. 133, pp. 93-100, 1988.

[23] E. K. Zavadskas, Z. Turskis, T. Vilutienè, and N. Lepkova, "Integrated group fuzzy multi-criteria model: case of facilities management strategy selection," Expert Systems with Applications, vol. 82, pp. 317-331, 2017.

[24] M. Abu-Saleem, "On fuzzy fundamental group and their foldings," European Journal of Scientific Research, vol. 148, pp. 368-376, 2018.

[25] G. Haçat, "On fuzzy retract of a fuzzy loop space," International Journal of Mathematics Trends and Technology, vol. 65, no. 2, pp. 73-82, 2019.

[26] S. A. Robertson, "Isometric folding of Riemannian manifolds," Proceedings of the Royal Society of Edinburgh: Section A Mathematics, vol. 79, no. 3-4, pp. 275-284, 1978.

[27] M. El-Ghoul, "Folding of fuzzy graphs and fuzzy spheres," Fuzzy Sets and Systems, vol. 58, no. 3, pp. 355-363, 1993.

[28] M. El-Ghoul and H. M. Shamara, "Folding of some types of fuzzy manifolds and their retractions," Fuzzy Sets and Systems, vol. 97, no. 3, pp. 387-391, 1998.

[29] A. E. El-Ahmady, "Fuzzy folding of fuzzy horocycle," Rendiconti Del Circolo Matematico Di Palermo, vol. 53, no. 3, pp. 443-450, 2004.

[30] A. E. El-Ahmady and K. Al-Onemi, "On fuzzy spheres in fuzzy Lobachevsky space and its retractions," Indian Journal of Science and Technology, vol. 6, no. 4, pp. 4243-4248, 2013.

[31] A. E. El-Ahmady and A. El-Araby, "On fuzzy spheres in fuzzy Minkowski space," Nuovo Cimento Della Societa Italiana Di Fisica B, vol. 125, no. 10, pp. 1153-1160, 2010.

[32] P. Zhou, L. Zhang, S. Zhang, and A. F. Alkhateeb, "Observerbased adaptive fuzzy finite-time control design with prescribed performance for switched pure-feedback nonlinear systems," IEEE Access, vol. 9, pp. 69481-69491, 2021.

[33] G. M. Webb, "Similarity considerations and conservation laws for magneto-static atmospheres," Solar Physics, vol. 106, no. 2, pp. 287-313, 1986.

[34] A. E. El-Ahmady and M. Abu-Saleem, "Homology groups and foldings on Buchdahi space," Indian Journal of Science and Technology, vol. 6, no. 6, pp. 5249-5256, 2013.

[35] A. E. El-Ahmady and M. Abu-Saleem, "Retractions of chaotic Buchdahi space and their chaotic fundamental groups," Indian Journal of Science and Technology, vol. 6, no. 8, pp. 5155-5162, 2013. 